OPEN ACCESS

Edited by:

Brahm Segal,

University at Buffalo, United States

Reviewed by:

Hongbin Wang,

California Northstate University,

United States

Wei Chen,

Stanford University, United States

${ }^{*}$ Correspondence:

Maria Teresa Masucci

m.masucci@istitutotumori.na.it

Specialty section:

This article was submitted to

Cancer Immunity and Immunotherapy,

a section of the journal

Frontiers in Immunology

Received: 22 April 2020

Accepted: 30 June 2020

Published: 16 September 2020

Citation

Masucci MT, Minopoli M, Del Vecchio S and Carriero MV (2020) The

Emerging Role of Neutrophil

Extracellular Traps (NETs) in Tumor

Progression and Metastasis.

Front. Immunol. 11:1749.

doi: 10.3389/fimmu.2020.01749

\section{The Emerging Role of Neutrophil Extracellular Traps (NETs) in Tumor Progression and Metastasis}

\author{
Maria Teresa Masucci ${ }^{1 *}$, Michele Minopoli ${ }^{1}$, Silvana Del Vecchio ${ }^{2}$ and \\ Maria Vincenza Carriero ${ }^{1}$ \\ ${ }^{1}$ Neoplastic Progression Unit, Istituto Nazionale Tumori IRCCS "Fondazione G. Pascale", Naples, Italy, ${ }^{2}$ Department of \\ Advanced Biomedical Sciences, University of Naples "Federico II", Naples, Italy
}

Neutrophil Extracellular Traps (NETs) are net-like structures composed of DNA-histone complexes and proteins released by activated neutrophils. In addition to their key role in the neutrophil innate immune response, NETs are also involved in autoimmune diseases, like systemic lupus erythematosus, rheumatoid arthritis, psoriasis, and in other non-infectious pathological processes, as coagulation disorders, thrombosis, diabetes, atherosclerosis, vasculitis, and cancer. Recently, a large body of evidence indicates that NETs are involved in cancer progression and metastatic dissemination, both in animal models and cancer patients. Interestingly, a close correlation between cancer cell recruitment of neutrophils in the tumor microenvironment (Tumor Associated Neutrophils. TANs) and NET formation has been also observed either in primary tumors and metastatic sites. Moreover, NETs can also catch circulating cancer cells and promote metastasis. Furthermore, it has been reported that wake dormant cancer cells, causing tumor relapse and metastasis. This review will primarily focus on the pro-tumorigenic activity of NETs in tumors highlighting their ability to serve as a potential target for cancer therapy.

\footnotetext{
Keywords: NETosis, tumor associated neutrophils (TANs), neutrophil extracellular trap (NET), tumor microenvironment (TEM), tumors
}

\section{INTRODUCTION}

Neutrophil Extracellular Traps (NETs) are web-like structures, discovered by Volker Brinkmann and Arturo Zychlinsky in 2004, able to entrap bacteria fungi, protozoa, and virus (1). They are extruded by activated neutrophils and are composed of DNA fibers, histones, and antimicrobial proteins (2-4), in which pathogens are immobilized and exposed to a local high and lethal concentration of effector proteins (5). Zychlinsky's group has demonstrated that NETs kill bacteria, thus defining a new modality of antimicrobial innate response (1).

Besides their role as a host defense mechanism, NETs play a pivotal role in non-infectious conditions, such as systemic lupus erythematosus (6), rheumatoid arthritis, cystic fibrosis $(7,8)$, and psoriasis (9). They are also involved in other pathological processes, such as coagulation disorders, thrombosis $(10)$, diabetes $(11,12)$, atherosclerosis (13), vasculitis (14), wound healing (12), and 
periodontitis $(15,16)$. Recently, several studies have investigated the role of NETs in tumors and reported their involvement in cancer immunoediting (3), progression (3, 17-19), metastatic spread (20), and cancer associated thrombosis (20). NETs can exert an anti-tumor or pro-tumor activity. NET components like myeloperoxidase (MPO) (21), proteinases $(20,22)$, and histones (23) can kill a tumor, inhibit tumor growth, and metastasis (21). NET proteinases can also degrade the extracellular matrix, promoting cancer cell extravasation, and metastasis $(20,22)$.

Furthermore, previous studies showed the ability of NETs to entrap and serve as an adhesion substrate for cancer cells $(24,25)$ thus promoting metastatic dissemination. NETs have been identified in peripheral blood and tumor specimens from animal models and cancer patients (26), but their role has not been completely elucidated. Moreover, circulating NETs are supposed to cause organ damage in cancer patients, similar to those occurring in auto-immune diseases (27), and their ability to awake dormant cancer cells has also been reported (28).

This review will primarily focus on the pro-tumorigenic activity of NETs in tumors, highlighting their ability to serve as a potential target for cancer therapy.

\section{NETosis}

The process by which neutrophils extrude NETs is known as NETosis. Different signals and mechanisms are involved in NET formation (29-31). Grayson and Kaplan suggested that the production of Reactive Oxygen Species (ROS), migration to the nucleus of neutrophil elastase (NE) and MPO, histone modification, and de-condensation are the pivotal events of NETosis (29). Sørensen and Borregaard proposed that NET formation pathways in vitro and in vivo could be distinct since the citrullination of histones by peptidyl arginine deiminase 4 (PAD4) and the consequent unwrapping of nucleosome are central events of NET formation in vivo $(32,33)$.

NETosis is considered a type of cell death, different from apoptosis and necrosis characterized by the release of decondensed chromatin and granular contents to the extracellular space (34). NETosis usually needs the stimulation of neutrophils and the generation of ROS by NADPH oxidase $(29,35,36)$. NETs generated from mitochondrial DNA are described (37) being the amount of mtDNA 100,000 times smaller than nuclear DNA because mitochondria are very few in neutrophils, but whether this difference plays any role, needs to be investigated further (38).

In addition to infectious and non-infectious diseases, NETs formation has been described also in the tumor microenvironment $(39,40)$. Cancer cell granulocytecolony-stimulating factor (G-CSF) and endothelial IL-8 are considered the main triggers of NETosis in tumors $(41,42)$. The over-expression of G-CSF in cancer causes a higher number of neutrophils in the blood, ROS generation, and subsequent NETosis $(43,44)$. Gupta et al. demonstrated that activated Endothelial Cells (ECs), co-cultured with neutrophils, can promote the NET formation, partially mediated by IL-8 (42). Finally, pancreatic cancer ( $\mathrm{PaCa}$ ) cells were shown to stimulate NETosis, directly or via PaCa-primed platelets (45).

\section{PRESENCE AND ROLE OF NETs IN TUMORS}

NETs have been identified in the blood and tumors of both animal models and patients where they may serve as pro- or antitumoral factors, depending on the status of the immune system or tumor microenvironment $(20,31)$. In particular, the correlation between NET formation, tumorigenesis, tumor progression, and metastasis has been investigated and there have been reports of the direct activity of NETs on tumor cell proliferation, through their proteases or activating signals $(3,19,20,46)$. Furthermore, the ability of cancer cells to promote NETosis in vivo and in vitro (41) and the presence of Tumor Associated Neutrophils (TANs) in the tumor microenvironment, as well as their association with NET formation, have been demonstrated (41, 47-49).

In the next two sections, we provide an overview of studies on the role of NETs in tumor models and cancer patients.

\section{NETs in Tumor Models}

Several studies showed that cancer cells can induce the release of NETs by neutrophils. In chronic myelogenous leukemia, mammary, and lung carcinoma mouse models, circulating neutrophils were more prone to release NETs than those of healthy animals. The high tendency of neutrophils to form NETs in these animals correlated to cancer systemic effects on the host $(43,50)$.

In two transgenic mouse tumor models, RIP1-Tag2 of insulinoma and MMTV-PyMT of breast cancer, vascular alterations, and enhanced neutrophil infiltration in the heart and kidneys, resembling tumor-induced systemic damage of cancer patients, were observed. Moreover, platelet-neutrophil complexes, indicative of NETs formation were found in the kidneys. DNase treatment completely restored kidney vascular function to levels seen in healthy mice, suggesting the causal role of NETs in the impaired vascular function of tumorbearing mice (27). In pancreatic adenocarcinoma (PDA), NETs and platelets have been demonstrated to play a pivotal role in blood hypercoagulability, resulting in an increased risk of venous thromboembolism, and in cancer-associated thrombosis, both in an orthotopic PDA model in C57/Bl6 mice and patients (51).

An in vitro study showed that a pancreatic cancer cell line (AsPC-1) induced the formation of NETs in a dose-dependent manner (17) and the major role in NETosis was attributed to protein components of AsPC-1 conditioned medium (CM). In the same study, NETs were shown to enhance the endogenous thrombin potential (ETP) of normal plasma and induce cancer cell migration, invasion, and angiogenesis (17). In another in vitro study, it was demonstrated that Lewis Lung Carcinoma (LLC) extracellular RNAs (exRNA) cause the formation of NETs (52). 


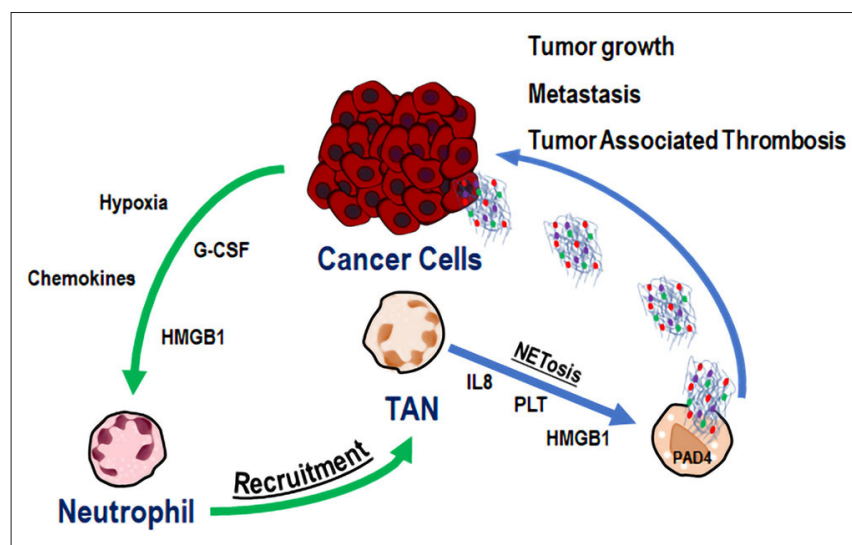

FIGURE 1 | Cancer cell, TAN, and NET co-operation in tumor progression and metastasis. Cancer cells recruit neutrophils to tumor microenvironment through several signals (green arrows). In tumor microenvironment Tumor Associated Neutrophils (TANs) are activated to release Neutrophil Extracellular Traps (NETs) (blue arrows), able to promote tumor growth, tumor progression, metastasis, and tumor associated thrombosis.

Furthermore, spontaneous intestinal tumorigenesis correlates with the accumulation of low-density neutrophils with a protumorigenic N2 phenotype, upregulation of complement C3a receptor, and NET formation (53).

The role of NETs in promoting tumor metastasis seems to be very important, and it has been reported that NETs can entrap circulating tumor cells (CTCs) thus favoring the metastatic dissemination. In a tumor murine model, mimicking the postoperative inflammatory environment, adhesion of cancer cells to NETs was demonstrated, $\beta 1$-integrin expression was upregulated and the expression of $\beta 1$-integrin on both cancer cells and NETs was essential for the adhesion of CTCs to NETs, in vitro, and in vivo. DNase treatment abrogated cancer cell adhesion to NETs (54). In a mouse model, NET formation and entrapment of circulating lung tumor cells caused a higher number of hepatic micro-metastasis (55). Finally, NETs were able to promote the development and progression of liver metastases after surgical stress (56).

Another issue that has been addressed is the close interaction between cancer cells, TANs, and NETs (Figure 1). Recently, Tohme et al. showed that NETs promoted tumor cell growth by enhancing their mitochondrial functions. Moreover, subcutaneous implanted tumors grew faster in WT than in PAD4-KO mice and liver metastases were fewer in PAD4KO compared to WT mice. Similar results were obtained by intra-peritoneal administration of DNase to WT mice. The immunofluorescent staining of tumor tissues from PAD4-KO tumors showed a very low neutrophil infiltration as compared to that of WT mice. Neutrophil recruitment by conditioned media from hypoxic cancer cells was observed in vitro and was mediated by high levels of chemokines and HMGB1, also able to generate NETs within the tumor microenvironment. Altogether, these data highlight the centrality of neutrophil recruitment and NET formation for tumor growth and progression (49).
In another interesting study, Park et al. highlighted the close relationship between metastatic cancer cells, neutrophil recruitment, and NET formation. They showed that metastatic breast cancer cells stimulate neutrophils to form metastasissupporting NETs. CXCL1 was able to mediate neutrophil recruitment into tumors from mice orthotopically transplanted with 4T1 (metastatic) and 4T07 (non-metastatic) murine breast cancer cells. More neutrophils were found in primary $4 \mathrm{~T} 1$ tumors than in 4T07 tumors and the reduction of CXCL1 in 4T1 cells lowered neutrophil infiltration into tumor lesions. By immunofluorescent staining for NETs of lung tissue sections from mice specimens, the NET formation was shown to occur just after 4T1 cell tail vein injection. Moreover, G-CSF makes metastatic cancer cells form NETs at sites of dissemination; and anti-GCSF blocking antibodies highly reduce the ability of $4 \mathrm{~T} 1$ cells to induce NETosis, in a trans-well chamber assay (41).

Besides all the previously described functions, NETs "wakeup" dormant tumor cells and cause metastasis. Recently, Albrengues et al. (28) demonstrated a clear role of NETs in tumor relapsing. In mice, the mechanism by which the dormant cells wake-up and produce metastases was demonstrated. Chronic lung inflammation caused by tobacco smoke or nasal instillation of LPS promoted lung metastases, due to the formation of NETs and suppressed in vitro and in vivo by digesting NET-DNA with DNase. Moreover, NETs bind the extracellular matrix and provoke laminin cleavage and remodeling, with the exposition of a new epitope that awakes the dormant cell proliferation, through integrin activation and FAK/ERK/MLCK/YAP signaling.

Different techniques could be used for the detection of adhesive interactions between NETs and tumor cells. Monti et al. (24) demonstrated that various cancer cell lines (HT1080, U87 MG, H1975, DU 145, PC-3, and A-431) were able to adhere in vitro to neutrophil-like cells derived NETs, via $\alpha 5 \beta 1, \alpha v \beta 3$, and $\alpha v \beta 5$ integrins expressed on the cell surface. An excess of cyclic RGD peptide inhibited cancer cell adhesion to NETs, at a rate similar to that obtained with DNase treatment. This approach can be used to screen cancer cells expressing RGD-binding integrins for their adhesion capability to NETs.

\section{NETs in Cancer Patients}

NETs have been correlated to cancer progression and metastatic spread also in human tumors. Levels of NETs in plasma of patients with different types of tumors including lung, pancreatic, and bladder cancer, were found to be higher than in healthy controls (57). In lung cancer patients, Li et al. demonstrated the presence of NETs in lung tissues, peripheral blood, and sputum (52). Moreover, in colorectal cancer patients, the levels of NETs produced by neutrophils after in vitro stimulation were significantly higher than in the healthy controls and associated with a poor clinical outcome (58).

Park et al. demonstrated the presence of NETs in human breast cancer by immuno-fluorescence staining. NETs were detected in metastatic lung lesions with the highest percentage of positivity in triple-negative tumors (41). Similarly, in histopathological samples of colorectal liver metastases from patients who underwent elective curative resection, Tohme et al. found a higher presence of TANs and NETs compared to normal liver. 
Also, tumor tissue expressed high levels of citrullinated histones, indicative of NETosis. Furthermore, preoperative levels of MPODNA, a reliable marker for systemic NETosis (56), were higher in patient serum than in healthy controls and were associated with poor DFS and OS. Thus, MPO-DNA serum levels could represent a possible prognostic biomarker in these patients (49).

The relationship between TANs and NET formation in patient tumors and its role in tumor immuno-editing has been investigated particularly in pediatric Ewing sarcoma. BergerAchituv et al. demonstrated the presence of TANs in diagnostic biopsies of Ewing Sarcoma pediatric patients. In two of these, NETs are derived from TANs activation. These two patients presented metastases and early relapse after high doses of chemotherapy, suggesting a possible role of NETs in Ewing Sarcoma progression (3).

The importance of NETs in cancer has been also highlighted by the relation of NETs with alteration of coagulation in tumor patients. It has been shown that NETs stimulate cancerassociated thrombosis in tumors, a phenomenon associated with a very bad prognosis in patients $(18,19)$. The circulating levels of NETs were measured in HCC patients, by evaluating their markers (DNA-histone complex, double-stranded DNA, neutrophil elastase). Similarly, contact system activation markers were measured. NETs and contact system activation markers were higher in HCC patients than in healthy controls (59). Jung et al. measured the circulating levels of NETs and hypercoagulability markers in patients with pancreatobiliary malignancies and found a correlation between high levels of NET markers and hypercoagulability markers (17). Moreover, higher levels of plasma Citrullinated histone $\mathrm{H} 3$ (H3Cit) were observed in cancer patients in advanced stages, compared to those of a healthy individual, and neutrophils from cancer patients showed a higher content of H3Cit than others. Furthermore, H3Cit in plasma of cancer patients does correlate with NE, MPO, IL-6, and IL-8, all activators of NETosis $(56,60)$.

\section{NETS AS POTENTIAL TARGETS IN CANCER THERAPY}

NETs can be regarded as promising therapeutic targets in cancer (Figure 2). Taking into account the role of NETs in enhancing the metastatic potential of cancer cells, it could be advantageous to inhibit NET formation and/or activity in tumors (41). Unfortunately, the ongoing clinical trials have not yet clarified the best modality to target NETs $(31,61)$. Gonzalez-Aparicio et al. have suggested the combination of immunotherapy strategies and drugs interfering with neutrophil chemoattraction and NET extrusion, as a possible treatment of cancer (39). Another issue to be considered is the actual lack of markers that can predict a good response to NETs interfering therapy in patients. The only available markers of NET formation are H3Cit and MPO-DNA, which may have prognostic value in cancer patients $(56,60)$. The detection of NETs in tumor biopsies and the expression of GCSF in human tumor samples were proposed as patient selection criteria for NET targeting therapies, but these studies did not provide conclusive evidence $(41,62)$. The semi-quantitative method for the identification of NETs in body fluids, based on the association of DNA with MPO, needs more investigation (63). The majority of experimental and clinical studies targeting NETs have been accomplished in pathologies other than cancer, such as autoimmune and pulmonary diseases or complications of autoimmune conditions, as reviewed by Jorch and Kubes (64).

In Systemic lupus erythematosus (SLE), it has been demonstrated that the serum endonuclease DNase is essential to degrade chromatin within NETs. Moreover, in some SLE patients, DNase dis-functions cause very severe kidney injuries, underlining the importance of a good balance in NET formation and degradation (65). Therefore, considering that the DNase can disrupt NET structure, NETs could represent a good target for DNase therapy (65). Based on this evidence, DNase has been tested in experimental cancer models. For instance, DNase treatment decreased disease burden in breast cancer mouse models (27). Moreover, in a mouse model of intra-abdominal sepsis mimicking cancer post-operative inflammatory environment, NETs trapping of circulating tumor cells and adhesion was abrogated, in vivo, by DNase (54). The systemic administration of DNase was also shown to slightly reduce experimental metastasis in a mouse model of metastatic lung cancer (55). On the contrary, DNase coated nanoparticles exerted a higher effect, due to the stabilization of DNase linked to nanoparticles in blood. DNase nanoparticles digested NETs in vitro and block breast cancer lung metastasis formation, in vivo, whereas they did not affect primary tumor growth $(41,62)$. These results in animal models stimulate the use of DNase in human cancer, with no toxicity in clinical studies. DNase treatment was approved by the FDA for cystic fibrosis patients, suffering from excessive NET release in their lungs (66) to dissolve dense mucus (4). Inhibitors of molecules involved in NETosis, and interfering with NET formation, used in non-cancer pathologies, could be tested in cancer patients. Among these, NE inhibitors, used in Chronic Obstructive Pulmonary Disease and inhibitors of PDA4 could improve the clinical outcome of cancer patients (4), even if the commercially available PAD4 inhibitors $\mathrm{Cl}$-amidine have short serum half-lives (67). Domingo-Gonzalez et al. demonstrated that $\mathrm{PGE}_{2}$ via EP2 or EP4, negatively regulates NETosis, both in mice and in patients transplanted with hematopoietic stem cells, thus revealing another physiologic substance able to antagonize NET formation (68). In another study (17), PGE1 was shown to inhibit both cancer cell- and PMA- induced NET formation, probably by the induction of intracellular cAMP and the reduction of intracellular calcium ions, necessary to form NETs (69). In the same study, anti-thrombin significantly reduced NET formation induced by cancer cells (17). Furthermore, the NET inhibitor chloroquine reduced blood platelet aggregation, circulating tissue factor, and decreasing hypercoagulability in tumor bearing mice. Interestingly, the same effects were identified in patients (51). In a recent review, Hector Salazar-Gonzalez and colleagues examined the role of NETs in renal diseases, highlighting the necessity to block NET formation to interfere with the pathogenesis of inflammatory and non-inflammatory diseases, including cancer (70). Finally, beyond the role of NETs as a possible therapeutic target in cancer, a direct therapeutic role 

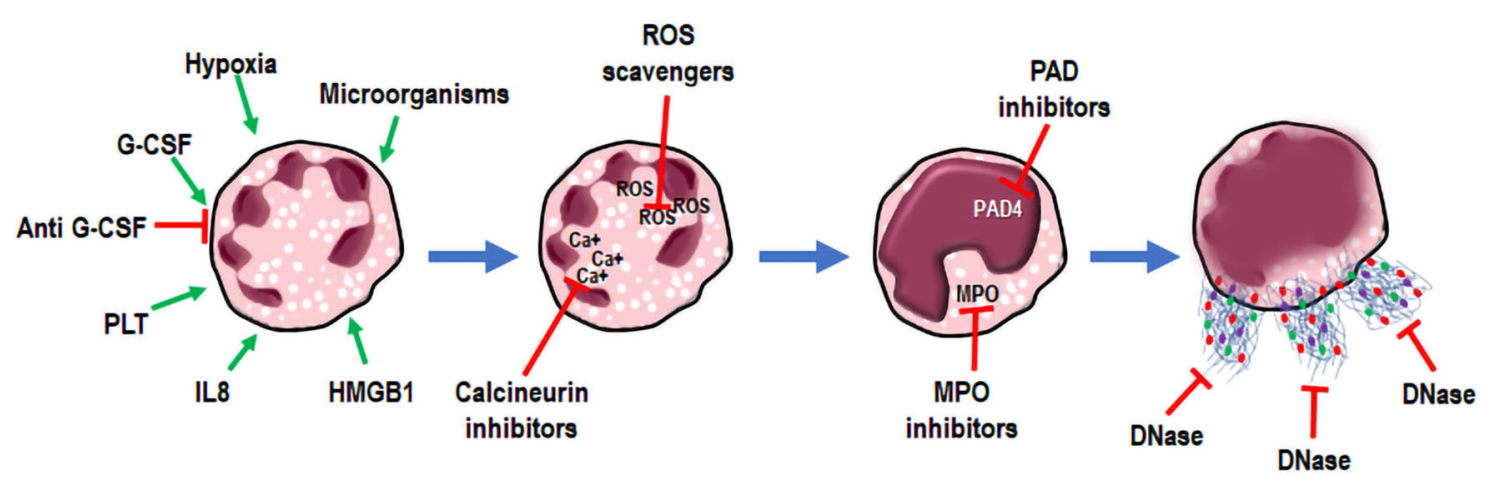

FIGURE 2 | NETosis. NETs as targets for therapy. Neutrophil stimulation (green arrows) results in NETosis and extrusion of Neutrophil Extracellular Traps (NETs) (blue arrows). During these processes, targets for therapies have been postulated, and interfering drugs (red arrows) have been already used in clinical practice or are under investigation in vivo.

of NETs has been reported in bladder cancer. NETs induced by Bacillus Calmette-Guerin (BCG) in the early stage of immunotherapy play an immune-regulatory role, by recruiting $\mathrm{T}$ cells and monocytes-macrophages, thus preventing tumor growth. Moreover, BCG induced NETs cause apoptosis and cell-cycle arrest, and inhibit cancer cell migration (71). Recently, the same group demonstrated that an immune reaction involving neutrophils is caused by BCG intravescical BCG infusion in the early stages of the treatment. BCG induces NET release in a time dependent manner, requiring ROS, being MEK, p38, PI3K, and PKC necessary to regulate the all process.

\section{CONCLUSIONS AND FUTURE DIRECTIONS}

The close cooperation between cancer cells, TANs, and NET formation in the tumor microenvironment highlights the function of NETs in cancer progression and metastasis. NETs are able to wake up dormant cancer cells, promoting cancer relapse and metastasis. In addition, they are also able to entrap circulating cancer cells and enhance metastasis spread.

Further studies are needed to investigate the potential role of NETs as targets for cancer therapy and to evaluate the possibility of pharmacologically interfering with NET formation and destruction, as it has been accomplished in autoimmune diseases. Promising results were obtained in tumor models whereas in cancer patients recent studies did not provide

\section{REFERENCES}

1. Brinkmann V, Reichard U, Goosmann C, Fauler B, Uhlemann Y, Weiss DS, et al. Neutrophil extracellular traps kill bacteria. Science. (2004) 303:1532-5. doi: 10.1126/science.109 2385

2. Dabrowska D, Jabłońska E, Garley M, Ratajczak-Wrona W, Iwaniuk A. New aspects of the biology of neutrophil extracellular traps. Scand J Immunol. (2016) 84:317-22. doi: 10.1111/sji.12494 conclusive evidence of the efficacy of this approach. Indeed, it will be necessary to focus future research on investigating the regulation of NETosis and the balance between NET formation and destruction, in order to pharmacologically target NETs, without hampering immune functions. Thereafter, clinical trials testing the efficacy of NETs interfering drugs in cancer patients could be designed.

\section{AUTHOR CONTRIBUTIONS}

MTM and MC: conception of the work. MTM and MM: extensive literature search and manuscript drafting. MTM, $\mathrm{SD}$, and $\mathrm{MC}$ : critical revision of the work and final version approval. All authors contributed to the article and approved the submitted version.

\section{FUNDING}

This work was partly supported by Institutional Funds from the National Cancer Institute (IRCCS), Fondazione G. Pascale, and by a grant from Regione Campania SATIN Project 2018-2020.

\section{ACKNOWLEDGMENTS}

The authors deeply appreciate the technical assistance provided by Gioconda Di Carluccio in creating the cartoons included in this review. 
7. Martínez-Alemán SR, Campos-García L, Palma-Nicolas JP, Hernández-Bello R, González GM, Sánchez-González A. Understanding the entanglement: neutrophil extracellular traps (NETs) in cystic fibrosis. Front Cell Infect Microbiol. (2017) 7:104. doi: 10.3389/fcimb.2017.00104

8. Gray RD, Hardisty G, Regan KH, Smith M, Robb CT, Duffin R, et al. Delayed neutrophil apoptosis enhances NET formation in cystic fibrosis. Thorax. (2018) 73:134-44. doi: 10.1136/thoraxjnl-2017-210134

9. Hoffmann JHO, Enk AH. Neutrophil extracellular traps in dermatology: caught in the NET. J Dermatol Sci. (2016) 84:3-10. doi: 10.1016/j.jdermsci.2016.07.001

10. Fuchs TA, Brill A, Duerschmied D, Schatzberg D, Monestier M, Myers DD, et al. Extracellular DNA traps promote thrombosis. Proc Natl Acad Sci USA. (2010) 107:15880-5. doi: 10.1073/pnas.1005743107

11. Wang L, Zhou X, Yin Y, Mai Y, Wang D, Zhang X. Hyperglycemia induces neutrophil extracellular traps formation through an NADPH oxidase-dependent pathway in diabetic retinopathy. Front Immunol. (2018) 9:3076. doi: 10.3389/fimmu.2018.03076

12. Wong SL, Demers M, Martinod K, Gallant M, Wang Y, Goldfine AB, et al. Diabetes primes neutrophils to undergo NETosis, which impairs wound healing. Nat Med. (2015) 21:815-9. doi: 10.1038/nm.3887

13. Warnatsch A, Ioannou M, Wang Q, Papayannopoulos V. Inflammation. Neutrophil extracellular traps license macrophages for cytokine production in atherosclerosis. Science. (2015) 349:316-20. doi: 10.1126/science.aaa8064

14. Nakazawa D, Shida H, Tomaru U, Yoshida M, Nishio S, Atsumi T, et al. Enhanced formation and disordered regulation of NETs in myeloperoxidaseANCA-associated microscopic polyangiitis. J Am Soc Nephrol. (2014) 25:9907. doi: 10.1681/ASN.2013060606

15. White PC, Chicca IJ, Cooper PR, Milward MR, Chapple ILC. Neutrophil extracellular traps in periodontitis: a web of intrigue. J Dent Res. (2016) 95:26-34. doi: 10.1177/0022034515609097

16. Vitkov L, Hartl D, Minnich B, Hannig M. Janus-faced neutrophil extracellular traps in periodontitis. Front Immunol. (2017) 8:1404. doi: 10.3389/fimmu.2017.01404

17. Jung HS, Gu J, Kim J-E, Nam Y, Song JW, Kim HK. Cancer cell-induced neutrophil extracellular traps promote both hypercoagulability and cancer progression. PLoS ONE. (2019) 14:e0216055. doi: 10.1371/journal.pone.0216055

18. Demers M, Wagner DD. NETosis: a new factor in tumor progression and cancer-associated thrombosis. Semin Thromb Hemost. (2014) 40:27783. doi: 10.1055/s-0034-1370765

19. Cools-Lartigue J, Spicer J, Najmeh S, Ferri L. Neutrophil extracellular traps in cancer progression. Cell Mol Life Sci. (2014) 71:4179-94. doi: 10.1007/s00018-014-1683-3

20. Homa-Mlak I, Majdan A, Mlak R, Małecka-Massalska T. Metastatic potential of NET in neoplastic disease. Postepy Hig Med Dosw. (2016) 70:88795. doi: 10.5604/17322693.1216275

21. Saffarzadeh M, Juenemann C, Queisser MA, Lochnit G, Barreto G, Galuska SP, et al. Neutrophil extracellular traps directly induce epithelial and endothelial cell death: a predominant role of histones. PLoS ONE. (2012) 7:e0032366. doi: 10.1371/journal.pone.0032366

22. Cedervall J, Hamidi A, Olsson A-K. Platelets, NETs and cancer. Thromb Res. (2018) 164:S148-52. doi: 10.1016/j.thromres.2018.01.049

23. Schedel F, Mayer-Hain S, Pappelbaum KI, Metze D, Stock M, Goerge T, et al. Evidence and impact of neutrophil extracellular traps in malignant melanoma. Pigment Cell Melanoma Res. (2020) 33:63-73. doi: 10.1111/pcmr.12818

24. Monti M, De Rosa V, Iommelli F, Carriero MV, Terlizzi C, Camerlingo R, et al. Neutrophil extracellular traps as an adhesion substrate for different tumor cells expressing RGD-binding integrins. Int J Mol Sci. (2018) 19:2350. doi: 10.3390/ijms19082350

25. Kanamaru R, Ohzawa H, Miyato H, Yamaguchi H, Hosoya Y, Lefor AK, et al. Neutrophil extracellular traps generated by low density neutrophils obtained from peritoneal lavage fluid mediate tumor cell growth and attachment. $J$ Vis Exp. (2018) 138:58201. doi: 10.3791/58201

26. Monti M, Iommelli F, De Rosa V, Carriero MV, Miceli R, Camerlingo $\mathrm{R}$, et al. Integrin-dependent cell adhesion to neutrophil extracellular traps through engagement of fibronectin in neutrophil-like cells. PLoS ONE. (2017) 12:e0171362. doi: 10.1371/journal.pone.0171362
27. Cedervall J, Zhang Y, Huang H, Zhang L, Femel J, Dimberg A, et al. Neutrophil extracellular traps accumulate in peripheral blood vessels and compromise organ function in tumor-bearing animals. Cancer Res. (2015) 75:2653-62. doi: 10.1158/0008-5472.CAN-14-3299

28. Albrengues J, Shields MA, Ng D, Park CG, Ambrico A, Poindexter $\mathrm{ME}$, et al. Neutrophil extracellular traps produced during inflammation awaken dormant cancer cells in mice. Science. (2018) 361:eaao4227. doi: 10.1126/science.aao4227

29. Grayson PC, Kaplan MJ. At the bench: neutrophil extracellular traps (NETs) highlight novel aspects of innate immune system involvement in autoimmune diseases. J Leukoc Biol. (2016) 99:253-64. doi: 10.1189/jlb.5BT0615-247R

30. Remijsen Q, Kuijpers TW, Wirawan E, Lippens S, Vandenabeele P, Vanden Berghe T. Dying for a cause: NETosis, mechanisms behind an antimicrobial cell death modality. Cell Death Differ. (2011) 18:5818. doi: 10.1038/cdd.2011.1

31. Saffarzadeh M. Neutrophil extracellular traps as a drug target to counteract chronic and acute inflammation. Curr Pharm Biotechnol. (2018) 19:1196202. doi: 10.2174/1389201020666190111164145

32. Sørensen OE, Borregaard N. Neutrophil extracellular traps-the dark side of neutrophils. J Clin Invest. (2016) 126:1612-20. doi: 10.1172/JCI84538

33. Wang Y, Li M, Stadler S, Correll S, Li P, Wang D, et al. Histone hypercitrullination mediates chromatin decondensation and neutrophil extracellular trap formation. J Cell Biol. (2009) 184:205-13. doi: $10.1083 /$ jcb.200806072

34. Lin AM, Rubin CJ, Khandpur R, Wang JY, Riblett M, Yalavarthi S, et al. Mast cells and neutrophils release IL-17 through extracellular trap formation in psoriasis. J Immunol. (2011) 187:490-500. doi: 10.4049/jimmunol.1100123

35. Fuchs TA, Abed U, Goosmann C, Hurwitz R, Schulze I, Wahn V, et al. Novel cell death program leads to neutrophil extracellular traps. J Cell Biol. (2007) 176:231-41. doi: 10.1083/jcb.200606027

36. Yousefi S, Gold JA, Andina N, Lee JJ, Kelly AM, Kozlowski E, et al. Catapultlike release of mitochondrial DNA by eosinophils contributes to antibacterial defense. Nat Med. (2008) 14:949-53. doi: 10.1038/nm.1855

37. Varjú I, Kolev K. Chapter 2: Fibrinolysis at the interface of thrombosis and inflammation - the role of neutrophil extracellular traps. In: Kolev K, editor. Fibrinolysis and Thrombolysis (2014). p. 31-59. doi: 10.5772/57259

38. Brinkmann V, Zychlinsky A. Neutrophil extracellular traps: is immunity the second function of chromatin? J Cell Biol. (2012) 198:773-83. doi: 10.1083/jcb.201203170

39. Gonzalez-Aparicio M, Alfaro C. Influence of interleukin-8 and neutrophil extracellular trap (NET) formation in the tumor microenvironment: is there a pathogenic role? J Immunol Res. (2019) 2019:6252138. doi: 10.1155/2019/6252138

40. Snoderly HT, Boone BA, Bennewitz MF. Neutrophil extracellular traps in breast cancer and beyond: current perspectives on NET stimuli, thrombosis and metastasis, and clinical utility for diagnosis and treatment. Breast Cancer Res. (2019) 21:145. doi: 10.1186/s13058-019-1237-6

41. Park J, Wysocki RW, Amoozgar Z, Maiorino L, Fein MR, Jorns J, et al. Cancer cells induce metastasis-supporting neutrophil extracellular DNA traps. Sci Transl Med. (2016) 8:361ra138. doi: 10.1126/scitranslmed.aag1711

42. Gupta AK, Joshi MB, Philippova M, Erne P, Hasler P, Hahn S, et al. Activated endothelial cells induce neutrophil extracellular traps and are susceptible to NETosis-mediated cell death. FEBS Lett. (2010) 584:31937. doi: 10.1016/j.febslet.2010.06.006

43. Demers M, Krause DS, Schatzberg D, Martinod K, Voorhees JR, Fuchs TA, et al. Cancers predispose neutrophils to release extracellular DNA traps that contribute to cancer-associated thrombosis. Proc Natl Acad Sci USA. (2012) 109:13076-81. doi: 10.1073/pnas.1200419109

44. Avalos BR, Gasson JC, Hedvat C, Quan SG, Baldwin GC, Weisbart RH, et al. Human granulocyte colony-stimulating factor: biologic activities and receptor characterization on hematopoietic cells and small cell lung cancer cell lines. Blood. (1990) 75:851-7.

45. Abdol Razak N, Elaskalani O, Metharom P. Pancreatic cancer-induced neutrophil extracellular traps: a potential contributor to cancer-associated thrombosis. Int J Mol Sci. (2017) 18:487. doi: 10.3390/ijms18030487

46. Sangaletti S, Tripodo C, Vitali C, Portararo P, Guarnotta C, Casalini P, et al. Defective stromal remodeling and neutrophil extracellular traps in lymphoid 
tissues favor the transition from autoimmunity to lymphoma. Cancer Discov. (2014) 4:110-29. doi: 10.1158/2159-8290.CD-13-0276

47. Masucci MT, Minopoli M, Carriero MV. Tumor associated neutrophils. Their role in tumorigenesis, metastasis, prognosis and therapy. Front Oncol. (2019) 9:1146. doi: 10.3389/fonc.2019.01146

48. Powell DR, Huttenlocher A. Neutrophils in the tumor microenvironment. Trends Immunol. (2016) 37:41-52. doi: 10.1016/j.it.2015.11.008

49. Yazdani HO, Roy E, Comerci AJ, van der Windt DJ, Zhang $H$, Huang $\mathrm{H}$, et al. Neutrophil extracellular traps drive mitochondrial homeostasis in tumors to augment growth. Cancer Res. (2019) 79:562639. doi: 10.1158/0008-5472.CAN-19-0800

50. Demers M, Wagner DD. Neutrophil extracellular traps: a new link to cancerassociated thrombosis and potential implications for tumor progression. Oncoimmunology. (2013) 2:e22946. doi: 10.4161/onci.22946

51. Boone BA, Murthy P, Miller-Ocuin J, Doerfler WR, Ellis JT, Liang $\mathrm{X}$, et al. Chloroquine reduces hypercoagulability in pancreatic cancer through inhibition of neutrophil extracellular traps. BMC Cancer. (2018) 18:678. doi: 10.1186/s12885-018-4584-2

52. Li Y, Yang Y, Gan T, Zhou J, Hu F, Hao N, et al. Extracellular RNAs from lung cancer cells activate epithelial cells and induce neutrophil extracellular traps. Int J Oncol. (2019) 55:69-80. doi: 10.3892/ijo.2019.4808

53. Guglietta S, Chiavelli A, Zagato E, Krieg C, Gandini S, Ravenda PS, et al. Coagulation induced by C3aR-dependent NETosis drives protumorigenic neutrophils during small intestinal tumorigenesis. Nat Commun. (2016) 7:11037. doi: $10.1038 /$ ncomms 11037

54. Najmeh S, Cools-Lartigue J, Rayes RF, Gowing S, Vourtzoumis P, Bourdeau $F$, et al. Neutrophil extracellular traps sequester circulating tumor cells via $\beta 1$-integrin mediated interactions. Int J Cancer. (2017) 140:232130. doi: 10.1002/ijc.30635

55. Cools-Lartigue J, Spicer J, McDonald B, Gowing S, Chow S, Giannias B, et al. Neutrophil extracellular traps sequester circulating tumor cells and promote metastasis. J Clin Invest. (2013) 123:3446-58. doi: 10.1172/JCI67484

56. Tohme S, Yazdani HO, Al-Khafaji AB, Chidi AP, Loughran P, Mowen $\mathrm{K}$, et al. Neutrophil extracellular traps promote the development and progression of liver metastases after surgical stress. Cancer Res. (2016) 76:1367-80. doi: 10.1158/0008-5472.CAN-15-1591

57. Oklu R, Sheth RA, Wong KHK, Jahromi AH, Albadawi H. Neutrophil extracellular traps are increased in cancer patients but does not associate with venous thrombosis. Cardiovasc Diagn Ther. (2017) 7:S140-9. doi: 10.21037/cdt.2017.08.01

58. Richardson JJR, Hendrickse C, Gao-Smith F, Thickett DR. Neutrophil extracellular trap production in patients with colorectal cancer in vitro. Int J Inflam. (2017) 2017:4915062. doi: 10.1155/2017/4915062

59. Seo JD, Gu J-Y, Jung HS, Kim YJ, Kim HK. Contact system activation and neutrophil extracellular trap markers: risk factors for portal vein thrombosis in patients with hepatocellular carcinoma. Clin Appl Thromb Hemost. (2019) 25:1076029618825310. doi: 10.1177/1076029618825310

60. Thålin $C$, Lundström $S$, Seignez $C$, Daleskog $M$, Lundström A, Henriksson $\mathrm{P}$, et al. Citrullinated histone $\mathrm{H} 3$ as a novel prognostic blood marker in patients with advanced cancer. PLOS ONE. (2018) 13:e0191231. doi: 10.1371/journal.pone.0191231
61. Wu L, Saxena S, Awaji M, Singh RK. Tumor-associated neutrophils in cancer: going pro. Cancers (Basel). (2019) 11:564. doi: 10.3390/cancers11040564

62. Hollmén M, Karaman S, Schwager S, Lisibach A, Christiansen AJ, Maksimow $\mathrm{M}$, et al. G-CSF regulates macrophage phenotype and associates with poor overall survival in human triple-negative breast cancer. Oncoimmunology. (2016) 5:e1115177. doi: 10.1080/2162402X.2015.1115177

63. Koenen RR. Editorial: neutrophil extracellular traps as therapeutic targets for inflammatory disease. Am J Pharmacol Toxicol. 9:200-2. doi: 10.3844/ajptsp.2014.200.202

64. Jorch SK, Kubes P. An emerging role for neutrophil extracellular traps in noninfectious disease. Nat Med. (2017) 23:279-87. doi: 10.1038/nm.4294

65. Hakkim A, Fürnrohr BG, Amann K, Laube B, Abed UA, Brinkmann $\mathrm{V}$, et al. Impairment of neutrophil extracellular trap degradation is associated with lupus nephritis. Proc Natl Acad Sci USA. (2010) 107:98138. doi: 10.1073/pnas.0909927107

66. Quan JM, Tiddens HA, Sy JP, McKenzie SG, Montgomery MD, Robinson PJ, et al. A two-year randomized, placebo-controlled trial of dornase alfa in young patients with cystic fibrosis with mild lung function abnormalities. J Pediatr. (2001) 139:813-20. doi: 10.1067/mpd.2001.118570

67. Knight JS, Subramanian V, O’Dell AA, Yalavarthi S, Zhao W, Smith CK, et al. Peptidylarginine deiminase inhibition disrupts NET formation and protects against kidney, skin and vascular disease in lupus-prone MRL/lpr mice. Ann Rheum Dis. (2015) 74:2199-206. doi: 10.1136/annrheumdis-2014-205365

68. Domingo-Gonzalez R, Martínez-Colón GJ, Smith AJ, Smith CK, Ballinger $\mathrm{MN}$, Xia M, et al. Inhibition of neutrophil extracellular trap formation after stem cell transplant by prostaglandin E2. Am J Respir Crit Care Med. (2016) 193:186-97. doi: 10.1164/rccm.201501-0161OC

69. Shishikura K, Horiuchi T, Sakata N, Trinh D-A, Shirakawa R, Kimura T, et al. Prostaglandin E2 inhibits neutrophil extracellular trap formation through production of cyclic AMP. Br J Pharmacol. (2016) 173:31931. doi: $10.1111 / \mathrm{bph} .13373$

70. Salazar-Gonzalez H, Zepeda-Hernandez A, Melo Z, Saavedra-Mayorga DE, Echavarria R. Neutrophil extracellular traps in the establishment and progression of renal diseases. Medicina (Kaunas). (2019) 55:431. doi: 10.3390/medicina55080431

71. Liu K, Sun E, Lei M, Li L, Gao J, Nian X, et al. BCG-induced formation of neutrophil extracellular traps play an important role in bladder cancer treatment. Clin Immunol. (2019) 201:4-14. doi: 10.1016/j.clim.2019.02.005

Conflict of Interest: The authors declare that the research was conducted in the absence of any commercial or financial relationships that could be construed as a potential conflict of interest.

Copyright (C) 2020 Masucci, Minopoli, Del Vecchio and Carriero. This is an openaccess article distributed under the terms of the Creative Commons Attribution License (CC BY). The use, distribution or reproduction in other forums is permitted, provided the original author(s) and the copyright owner(s) are credited and that the original publication in this journal is cited, in accordance with accepted academic practice. No use, distribution or reproduction is permitted which does not comply with these terms. 